\title{
On multi-color partitions and the generalized Rogers-Ramanujan identities
}

\author{
Naihuan Jing * \\ Department of Mathematics \\ North Carolina State University \\ Raleigh, North Carolina 27695-8205, USA \\ jing@math.ncsu.edu \\ Kailash C. Misra ${ }^{\dagger}$ \\ Department of Mathematics \\ North Carolina State University \\ Raleigh, North Carolina 27695-8205, USA \\ misra@math.ncsu.edu \\ Carla D. Savage $\ddagger$ \\ Department of Computer Science \\ North Carolina State University \\ Raleigh, North Carolina 27695-8206, USA \\ savage@cayley.csc.ncsu.edu
}

November 29, 2000

\begin{abstract}
Basil Gordon, in the sixties, and George Andrews, in the seventies, generalized the Rogers-Ramanujan identities to higher moduli. These identities arise in many areas of mathematics and mathematical physics. One of these areas is representation theory of infinite dimensional Lie algebras, where various known interpretations of these identities have led to interesting applications. Motivated by their connections with Lie algebra representation theory, we give a new interpretation of a sum related to generalized Rogers-Ramanujan identities in terms of multi-color partitions.
\end{abstract}

\section{Introduction}

The celebrated Rogers-Ramanujan identities and their generalizations (see [G], An1]) have influenced current research in many areas of mathematics and physics (see An2, BeM]).

\footnotetext{
${ }^{*}$ Research supported in part by NSA grant MDA 904-97-1-0062 and NSF grant DMS-9701755 at MSRI.

${ }^{\dagger}$ Research supported in part by NSA grant MDA 904-00-1-0042.

${ }^{\ddagger}$ Research supported in part by NSF grant DMS9622772 and NSA grant MDA 904-00-1-0059
} 
These identities can be expressed as:

$$
\prod_{n \neq 0, \pm 2(\bmod 5)}\left(1-q^{n}\right)^{-1}=\sum_{n \geq 0} \frac{q^{n^{2}}}{(1-q)\left(1-q^{2}\right) \ldots\left(1-q^{n}\right)}
$$

and

$$
\prod_{n \neq 0, \pm 1(\bmod 5)}\left(1-q^{n}\right)^{-1}=\sum_{n \geq 0} \frac{q^{n^{2}+n}}{(1-q)\left(1-q^{2}\right) \ldots\left(1-q^{n}\right)} .
$$

Identities (1) and (2) have a natural combinatorial interpretation in terms of partitions, which was generalized by Gordon ([G], An1], Theorem 7.5). A partition of a positive integer $n$ is a finite, non-increasing sequence of positive integers, called parts, whose sum is $n$.

Theorem 1 [Gordon] For $M=2 k+1$ and $0<r \leq k$, the number of partitions of $n$ of the form $\left(\pi_{1}, \pi_{2}, \ldots, \pi_{l}\right)$, where $\pi_{j}-\pi_{j+k-1} \geq 2$ and at most $r-1$ of the parts are 1 is equal to the number of partitions of $n$ into parts not congruent to $0, r$, or $-r$ modulo $M$.

Setting $r=2$ and $M=5$ in Theorem 1 gives (1) and $r=1, M=5$ gives (2).

About twenty years ago it was observed that these identities play an important role in the representation theory of affine Lie algebras via its principal characters [LM]. In 1978 Lepowsky and Wilson [LW1] gave the first explicit realization of the affine Lie algebra $\widehat{\mathfrak{s l}}(2)$. This led to a new algebraic structure called the Z-algebra [LW2] which gave a formal foundation to study systematically the connection between affine Lie algebras and combinatorial identities. In particular, Lepowsky and Wilson [LW2, LW3, LW4] used the Z-algebra structure to construct the integrable highest weight representations of the affine Lie algebra $\widehat{\mathfrak{s l}}(2)$ and gave a $Z$-algebraic proof of the Rogers-Ramanujan identities and $Z$-algebraic interpretation of the generalized Rogers-Ramanujan identities.

One can also use (generalized) Rogers-Ramanujan identities to construct explicitly integrable representations of other affine Lie algebras. See for example [BM], [M1], [M2], Ma], [X] for these developments. In this connection the $Z$-operators still play an important role. These operators act on a certain space $\Omega(V)$ called the vacuum space associated with the representation space $V$ [LW3] and are parameterized by the set of roots of the associated simple Lie algebra $\mathfrak{g}$. However, on $V$ many of these $Z$-operators are scalar multiples of each other. For example, let us consider the affine Lie algebra $\hat{\mathfrak{g}}=\widehat{\mathfrak{s l}}(5)$ and its integrable highest weight representation $V(\lambda)$, with highest weight $\lambda=\Lambda_{0}+\Lambda_{2}$, where $\Lambda_{i}$ are the fundamental weights of the Lie algebra $\hat{\mathfrak{g}}$. The principal character of $V(\lambda)$ is

$$
\chi(V(\lambda))=F \prod_{n \geq 1, n \neq 0, \pm 3(\bmod 7)}\left(1-q^{n}\right)^{-1}
$$


where $F=\prod_{n \geq 1, n \neq 0 \text { mod } 5}\left(1-q^{n}\right)^{-1}$. In this case there are two independent families of $Z$-operators:

$$
Z(\beta, z)=\sum_{i \in \mathbb{Z}} Z(\beta, i) z^{-i}, \quad Z(\beta, i) \in \operatorname{End} V(\lambda)
$$

for $\beta=\beta_{1}$ and $\beta_{1}+\beta_{2}$, where $\left\{\beta_{1}, \beta_{2}, \beta_{3}, \beta_{4}\right\}$ are the simple roots of $\mathfrak{s t}(5)$ corresponding to the principal Cartan subalgebra $\mathfrak{a}$ (see M3]). In M3 $V\left(\Lambda_{0}+\Lambda_{2}\right)$ has been constructed using only one set of $Z$-operaters $Z\left(\beta_{1}, z\right)$ and Gordon's generalization of the Rogers-Ramanujan identities with $r=3$ and $M=7$ :

$$
\prod_{n \neq 0, \pm 3(\bmod 7)}\left(1-q^{n}\right)^{-1}=\sum_{n \geq 0} a(n) q^{n}
$$

where $a(n)$ denotes the number of partitions of $n$ such that the outer two of any three consecutive parts differ by at least 2 and at most two parts are 1 . However, from the representation theory point of view it would be more natural to construct the representation using both families of operators $Z\left(\beta_{1}, z\right)$ and $Z\left(\beta_{1}+\beta_{2}, z\right)$. It is expected that this would correspond to another expansion of the left-hand side of (3), namely

$$
\prod_{n \neq 0, \pm 3(\bmod 7)}\left(1-q^{n}\right)^{-1}=\sum_{n_{1} \geq n_{2} \geq 0} \frac{q^{n_{1}^{2}+n_{2}^{2}}}{(q)_{n_{1}-n_{2}}(q)_{n_{2}}}
$$

where we let $(a)_{n}=\prod_{k=0}^{n-1}\left(1-a q^{k}\right)$. The expansion (车) is a special case of Andrews' and Bressoud's analytic generalization of the Rogers-Ramanujan identities:

$$
\prod_{n \neq 0, \pm r(\bmod 2 k+s)}\left(1-q^{n}\right)^{-1}=\sum_{n_{1} \geq \ldots \geq n_{k-1} \geq 0} \frac{q^{n_{1}^{2}+n_{2}^{2}+\ldots+n_{k-1}^{2}+n_{r}+n_{r+1}+\ldots+n_{k-1}}}{(q)_{n_{1}-n_{2}} \ldots(q)_{n_{k-2}-n_{k-1}}\left(q^{2-s} ; q^{2-s}\right)_{n_{k-1}}},
$$

where $s=0,1$. Andrews ( An1], Theorem 7.8) derived this generalization for the case of odd modulus $(s=1)$ and Bressoud [Br1] for the case of even modulus $(s=0)$. In [FS] Feigin and Stoyanovsky used the representation of [LP] in the homogeneous gradation to give certain combinatorial interpretations of the multisum side of the generalized Rogers-Ramanujan identities. Later Georgiev Ge] and also Meurman and Primc (MP1], [MP2], [MP3] and [P]) related the sum sides of various generalized Rogers-Ramanujan type expressions to multi-color partitions by attaching colors to different roots in $Z$-algebra type constructions of the homogeneous irreducible highest weight representations of certain affine Lie algebras. It is clear from this work that the language of multi-color partitions are suitable for Zalgebraic constructions and interpretations of Rogers-Ramanujan type identities. The Zoperator constructions in the principal gradation motivated us to seek a new multi-color interpretation (corresponding to the parameters $n_{1}, n_{2}, \ldots, n_{k-1}$ in the sum side of (5)) of the generalized Rogers-Ramanujan identities.

It is well-known that the product side of (5) can also be written as

$$
\prod_{n \neq 0, \pm r(\bmod M)}\left(1-q^{n}\right)^{-1}=\frac{1}{(q)_{\infty}} \sum_{j=-\infty}^{\infty}(-1)^{j} q^{j[(M) j+M-2 r] / 2}
$$


a result which follows from the Jacobi Triple Product Identity. In this paper, we provide a combinatorial description of the sum side of (6) in terms of multi-color partitions $(k-1$ colors for identity (5)) with the hope that this will give new insights into the Z-operator constructions in the principal gradation. Our work builds on an interpretation of the sum side of (6) in terms of partitions with bounds on successive ranks due to Andrews [An3] and Bressoud [Br2]. Andrews and Bressoud showed that the sum side of (6) is the generating function for $\left|A_{n}(M, r)\right|$, where $A_{n}(M, r)$ is the set of all partitions of $n$ whose successive ranks lie in the interval $[-r+2, M-r-2]$ (to be discussed in more detail in Section 2). Our main theorem establishes a bijection between $A_{n}(M, r)$ and a family of multi-color partitions as described below.

Definition 1 For $t \geq 1$, a $t$-color partition of $n$ is a pair $\left(\alpha, c_{\alpha}\right)$ where $\alpha=\left(\alpha_{1}, \alpha_{2}, \ldots, \alpha_{l}\right)$ is a partition of $n$ and $c_{\alpha}$ is a function which assigns to each $i \in\{1,2, \ldots, l\}$ one of the colors $\{1,2, \ldots, t\}$ so that if $\alpha_{i}=\alpha_{i+1}$ then $c_{\alpha}(i) \leq c_{\alpha}(i+1)$. We say that $c_{\alpha}(i)$ is the color of the $i$-th part of $\alpha$.

For example, $\left(8_{2}, 8_{3}, 5_{1}, 4_{1}, 4_{2}, 4_{3}, 3_{2}, 2_{1}\right)$ is a 3 -color partition of 38 , where the subscript of a part denotes its color.

Our main theorem is stated below and proved in Section 2 as Theorem 3 .

Main Theorem For integers $r, M$, and $k$ satisfying $0<r \leq M / 2$ and $k=\lfloor M / 2\rfloor$, let $C_{n}(M, r)$ be the set of $k-1$-color partitions $\left(\alpha, c_{\alpha}\right)$ of $n$ satisfying the following three conditions. Let $\alpha=\left(\alpha_{1}, \alpha_{2}, \ldots, \alpha_{l}\right)$.

(i) (Initial Conditions) For $1 \leq i \leq l$,

$$
\alpha_{i}>\left\{\begin{array}{ll}
\left|2 c_{\alpha}(i)-r+1\right| & \text { if } \alpha_{i} \equiv r(\bmod 2) \\
\left|2 c_{\alpha}(i)-r\right| & \text { otherwise }
\end{array} .\right.
$$

(ii) (Color Difference Conditions) For $1 \leq i<l$,

$$
\alpha_{i}-\alpha_{i+1} \geq \begin{cases}2+\left|2\left(c_{\alpha}(i)-c_{\alpha}(i+1)\right)\right| & \text { if } \alpha_{i} \equiv \alpha_{i+1}(\bmod 2) \\ 2+\left|2\left(c_{\alpha}(i)-c_{\alpha}(i+1)\right)-1\right| & \text { if } \alpha_{i} \not \equiv \alpha_{i+1} \equiv r(\bmod 2) \\ 2+\left|2\left(c_{\alpha}(i)-c_{\alpha}(i+1)\right)+1\right| & \text { if } \alpha_{i+1} \not \equiv \alpha_{i} \equiv r(\bmod 2)\end{cases}
$$

(iii) ( Parity Condition on Last Color when $M$ is Even) For $1 \leq i \leq l$, if $M$ is even and $c_{\alpha}(i)=k-1$, the last color, then

$$
\alpha_{i} \not \equiv r \quad(\bmod 2) .
$$

Then

$$
\sum_{n=0}^{\infty}\left|C_{n}(M, r)\right| q^{n}=\sum_{n=0}^{\infty}\left|A_{n}(M, r)\right| q^{n}=\frac{1}{(q)_{\infty}} \sum_{j=-\infty}^{\infty}(-1)^{j} q^{j[(M) j+M-2 r] / 2}
$$


Our results suggest several interesting problems. Our motivating problem is to make use of the multi-color partition interpretation to construct natural realizations of integrable representations of affine Lie algebras, via a correspondence between the $k-1$ colors and the parameters $n_{1}, \ldots, n_{k-1}$ of (5). However, another intriguing problem is to establish a direct bijection between the multi-color partitions and the partitions counted by the sum side of the Rogers-Ramanujan identity (5), possibly through $A_{n}(M, r)$. We note some related work for the sum side. In [An4], Andrews gave a combinatorial interpretation of the sum side of (5) in terms of Durfee dissection partitions. Another result, due to Burge [Bu1, Bu2] and formulated in terms of lattice paths by Bressoud [Br3], interprets the sum side of (5) as the number of lattice paths of weight $n$ starting at $(0, k-r)$ which have no peak of height $k$ or greater. (Steps allowed in the lattice path are: $(x, y) \rightarrow(x+1, y+1) ;(x, 0) \rightarrow(x+1,0)$; and, if $y>0,(x, y) \rightarrow(x+1, y-1)$. The weight of a lattice path is the sum of the $x$-coordinates of its peaks.)

Although we have never seen a multi-color interpretation of (5), together with its conditions, made explicit, multi-color interpretations of other identities of the Rogers-Ramanujan type appear, for example, in [AA], [AB], Ge], ( MP1], [MP2], [MP3] and [P]). In Section 3 we note that ideas implicit in the papers $\mathrm{AA}$ and $[\mathrm{AB}$ give rise to an alternative multi-color interpretation of (5).

In Section 4, we give an example of a context in which the multi-color interpretation seems quite natural. In [FQ], Foda and Quano derive a finitization of a form of the generalized Rogers-Ramanujan identities. We show that the corresponding refinement for our multi-color partitions is simply an additional constraint on the size of the largest part.

\section{Multi-color partitions}

In this section, we give a new combinatorial interpretation of the product side of (5). Our main tool will be a combinatorial generalization of the Rogers-Ramanujan identities, due to George Andrews, which involves the successive ranks of a partition.

A partition $\pi=\left(\pi_{1}, \pi_{2}, \ldots, \pi_{l}\right)$ can be visualized by its Ferrers diagram, an array of dots, where $\pi_{i}$ is the number of left justified dots in the $i$ th row. The largest square subarray of dots in this diagram is the Durfee square and the Durfee square size, denoted by $d(\pi)$, is the length of a side. Flipping the diagram along its main diagonal, one obtains the dual diagram, associated with its dual partition $\pi^{\prime}=\left(\pi_{1}^{\prime}, \pi_{2}^{\prime}, \ldots, \pi_{\pi_{1}}^{\prime}\right)$, where $\pi_{i}^{\prime}$ is the

number of indices $j$ with $\pi_{j} \geq i$. The sequence of successive ranks of $\pi$ is the sequence $\left(\pi_{1}-\pi_{1}^{\prime}, \pi_{2}-\pi_{2}^{\prime}, \ldots, \pi_{d}-\pi_{d}^{\prime}\right)$, where $d=d(\pi)$.

Let $\pi$ be a partition of $n$ with successive ranks $r_{1}, r_{2}, \ldots, r_{d}$. For $1 \leq i \leq d=d(\pi)$, let

$$
\alpha_{i}=\pi_{i}+\pi_{i}^{\prime}-2(i-1)-1=\pi_{i}+\pi_{i}^{\prime}-2 i+1,
$$

i.e., $\alpha_{i}$ is the number of dots on the $i$-th "angle" of $\pi$. Denote by $\alpha(\pi)$ the partition with 


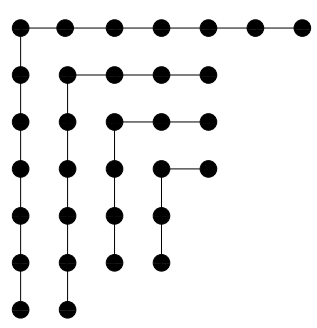

Figure 1: The Ferrers diagram of $\pi=(7,5,5,5,4,4,2)$ with successive ranks $0,-2,-1,-1$, and the four angles indicated, illustrating that $\alpha(\pi)=(13,9,6,4)$.

parts: $\alpha_{1}, \alpha_{2}, \cdots, \alpha_{d}$. Note that $\alpha(\pi)$ is a partition of $n$ such that

$$
\alpha_{i} \equiv r_{i}+1 \quad(\bmod 2) .
$$

(See Figure 1.)

Lemma 1 For $1 \leq i \leq d(\pi), \alpha_{i}>\left|r_{i}\right|$.

Proof. Since $i \leq d(\pi)$, we have $\pi_{i} \geq i$ and $\pi_{i}^{\prime} \geq i$, so

$$
\alpha_{i}=\pi_{i}+\pi_{i}^{\prime}-2 i+1=r_{i}+2 \pi_{i}^{\prime}-2 i+1 \geq r_{i}+2 i-2 i+1=r_{i}+1
$$

and

$$
\alpha_{i}=\pi_{i}+\pi_{i}^{\prime}-2 i+1=-r_{i}+2 \pi_{i}-2 i+1 \geq-r_{i}+2 i-2 i+1=-r_{i}+1
$$

Lemma 2 For $1 \leq i<d(\pi)$,

$$
\alpha_{i}-\alpha_{i+1} \geq 2+\left|r_{i}-r_{i+1}\right| .
$$

Proof. If $r_{i} \geq r_{i+1}$, then since $\pi_{i}^{\prime} \geq \pi_{i+1}^{\prime}$,

$$
\pi_{i}-\pi_{i+1} \geq \pi_{i}-\pi_{i+1}-\left(\pi_{i}^{\prime}-\pi_{i+1}^{\prime}\right)=r_{i}-r_{i+1} .
$$

Thus

$$
\alpha_{i}-\alpha_{i+1}=\pi_{i}-\pi_{i+1}+\pi_{i}^{\prime}-\pi_{i+1}^{\prime}+2 \geq r_{i}-r_{i+1}+2 .
$$

Similarly, If $r_{i} \leq r_{i+1}$, then since $\pi_{i} \geq \pi_{i+1}$,

$$
\pi_{i}^{\prime}-\pi_{i+1}^{\prime} \geq \pi_{i}^{\prime}-\pi_{i+1}^{\prime}-\left(\pi_{i}-\pi_{i+1}\right)=-r_{i}+r_{i+1} .
$$

Thus

$$
\alpha_{i}-\alpha_{i+1}=\pi_{i}^{\prime}-\pi_{i+1}^{\prime}+\pi_{i}-\pi_{i+1}+2 \geq-r_{i}+r_{i+1}+2 .
$$


Definition 2 Call a partition type 1 if successive parts differ by at least 2.

Corollary 1 For any partition $\pi, \alpha(\pi)$ is a type 1 partition.

Proof. By Lemma 2, $\alpha_{i}-\alpha_{i+1} \geq 2+\left|r_{i}-r_{i+1}\right| \geq 2$.

Remark 1 The number of partitions of $n$ with all successive ranks in $[0,1]$ is equal to the number of type 1 partitions of $n$. The map $\pi \rightarrow \alpha(\pi)$ is a bijection.

Remark 2 The number of partitions of $n$ with all successive ranks in $[1,2]$ is equal to the number of type 1 partitions of $n$ in which every part is larger than 1. The map $\pi \rightarrow \alpha(\pi)$ is again a bijection.

We can now state Andrews' generalization of the Rogers-Ramanujan identities. The theorem below was established by Andrews for odd moduli $M$ An3 and was generalized to even moduli by Bressoud [Br2].

Theorem 2 [Andrews-Bressoud] For integers $M, r$, satisfying $0<r \leq M / 2$, let $A_{n}(M, r)$ be the set of partitions of $n$ with all successive ranks in the interval $[-r+2, M-r-2]$. Then $\left|A_{n}(M, r)\right|$ is equal to the number of partitions of $n$ with no part congruent to $0, r$, or -r modulo $M$.

The smallest $M$ in the above theorem is 4 , as when $M=3$ and $r=1$, the interval $[-r+2, M-r-2]$ and the set $A_{n}(M, r)$ are both empty. To see that Theorem 2 generalizes the Rogers-Ramanujan identities, note that when $r=2$ and $M=5$, the theorem says that the number of partitions of $n$ with all ranks in $[0,1]$ is equal to the number of partitions of $n$ using no part congruent to 0, 2 or 3 modulo 5. By Remark 1 and Theorem 1, this is the first Rogers-Ramanujan identity (1). When $r=1, M=5$, the Andrews-Bressoud theorem says that the number of partitions of $n$ with all ranks in $[1,2]$ is equal to the number of partitions of $n$ using no part congruent to 0,1 or 4 modulo 5. By Remark 2 and Theorem 11, this is the second Rogers-Ramanujan identity (2).

In fact, what Andrews and Bressoud prove is:

$$
\sum_{n=0}^{\infty}\left|A_{n}(M, r)\right| q^{n}=\frac{1}{(q)_{\infty}} \sum_{j=-\infty}^{\infty}(-1)^{j} q^{j[(M) j+M-2 r] / 2}=\prod_{n \neq 0, \pm r(\bmod M)}\left(1-q^{n}\right)^{-1}
$$

where the first equality (the hard part) follows by a sieve argument and the second equality by application of the Jacobi Triple Product identity.

We now show that the partitions defined by Andrews' rank conditions are equivalent to certain classes of multi-color partitions. 
Theorem 3 For integers $r, M$, and $k$ satisfying $0<r \leq M / 2$ and $k=\lfloor M / 2\rfloor$, let $C_{n}(M, r)$ be the set of $k-1$-color partitions $\left(\alpha, c_{\alpha}\right)$ of $n$ satisfying the following three conditions. Let $\alpha=\left(\alpha_{1}, \alpha_{2}, \ldots, \alpha_{l}\right)$.

(i) (Initial Conditions) For $1 \leq i \leq l$,

$$
\alpha_{i}>\left\{\begin{array}{ll}
\left|2 c_{\alpha}(i)-r+1\right| & \text { if } \alpha_{i} \equiv r(\bmod 2) \\
\left|2 c_{\alpha}(i)-r\right| & \text { otherwise }
\end{array} .\right.
$$

(ii) (Color Difference Conditions) For $1 \leq i<l$,

$$
\alpha_{i}-\alpha_{i+1} \geq \begin{cases}2+\left|2\left(c_{\alpha}(i)-c_{\alpha}(i+1)\right)\right| & \text { if } \alpha_{i} \equiv \alpha_{i+1}(\bmod 2) \\ 2+\left|2\left(c_{\alpha}(i)-c_{\alpha}(i+1)\right)-1\right| & \text { if } \alpha_{i} \not \equiv \alpha_{i+1} \equiv r(\bmod 2) \\ 2+\left|2\left(c_{\alpha}(i)-c_{\alpha}(i+1)\right)+1\right| & \text { if } \alpha_{i+1} \not \equiv \alpha_{i} \equiv r(\bmod 2)\end{cases}
$$

(iii) (Parity Condition on Last Color when $M$ is Even) For $1 \leq i \leq l$, if $M$ is even and $c_{\alpha}(i)=k-1$, the last color, then

$$
\alpha_{i} \not \equiv r \quad(\bmod 2)
$$

Then the number of partitions in the two sets $A_{n}(M, r)$ and $C_{n}(M, r)$ are the same.

Proof. This follows from (8) once we establish a bijection between $A_{n}(M, r)$ and $C_{n}(M, r)$. Let $\pi$ be a partition of $n$ with ranks $r_{1}, r_{2}, \ldots r_{d(\pi)}$, and assume that all ranks lie in the interval $[-r+2, M-r-2]$. For $1 \leq i \leq d$, let $\alpha_{i}=\pi_{i}+\pi_{i}^{\prime}-2 i+1$, as before, and let $\alpha(\pi)$ be the partition of $n$ defined by $\alpha_{1}, \alpha_{2}, \ldots, \alpha_{d}$.

Color the parts of $\alpha$ as follows: for $1 \leq i \leq d$,

$$
c_{\alpha}(i)= \begin{cases}\left(r_{i}+r-1\right) / 2 & \text { if } \alpha_{i} \equiv r(\bmod 2) \\ \left(r_{i}+r\right) / 2 & \text { otherwise }\end{cases}
$$

We show that the $k-1$-color partition $\left(\alpha, c_{\alpha}\right)$ satisfies conditions (i)-(iii) of the theorem. Condition(i). By Lemma 1, $\alpha_{i}>\left|r_{i}\right|$ and from (9),

$$
r_{i}= \begin{cases}2 c_{\alpha}(i)-r+1 & \text { if } \alpha_{i} \equiv r(\bmod 2) \\ 2 c_{\alpha}(i)-r & \text { otherwise }\end{cases}
$$

Condition(ii). By Lemma 2, $\alpha_{i}-\alpha_{i+1} \geq 2+\left|r_{i}-r_{i+1}\right|$ and the conditions follow from (10). Condition(iii). If $M=2 k$ and $c_{\alpha}(i)=k-1$, then by definition of $c_{\alpha}$,

$$
r_{i} \in\{-r+2 k-2,-r+2 k-1\}=\{M-r-2, M-r-1\} .
$$


But $r_{i} \neq M-r-1$ since $r_{i} \in[-r+2, M-r-2]$. It follows then that $r_{i}=M-r-2$ and therefore that

$$
\begin{aligned}
\alpha_{i} & =\pi_{i}+\pi_{i}^{\prime}-2 i+1=r_{i}+2 \pi_{i}^{\prime}-2 i+1 \\
& =M-r-1+2\left(\pi_{i}^{\prime}-i\right) \equiv r+1 \quad(\bmod 2) .
\end{aligned}
$$

To show this is a bijection, given $r, M$, and $k$ satisfying the conditions of the theorem, let $\left(\alpha, c_{\alpha}\right)$ be a $k-1$ color partition of $n$, with $\alpha=\left(\alpha_{1}, \ldots, \alpha_{l}\right)$, satisfying (i), (ii), and (iii) of the theorem. We define an inverse map which sends $\alpha$ to a partition $\pi$ of $n$ with $d$ angles, where the $i$ th angle of $\pi$ has width $x_{i}$ defined by

$$
x_{i}= \begin{cases}\left(-r+2 c_{\alpha}(i)+\alpha_{i}+2\right) / 2 & \text { if } \alpha_{i} \equiv r(\bmod 2) \\ \left(-r+2 c_{\alpha}(i)+\alpha_{i}+1\right) / 2 & \text { otherwise }\end{cases}
$$

and height $y_{i}=\alpha_{i}-x_{i}+1$. We must verify that $\pi$ is a partition, i.e. that $x_{1}>x_{2}>$ $\ldots>x_{d} \geq 1$ and $y_{1}>y_{2}>\ldots>y_{d} \geq 1$ (clearly it has weight $n$ ), and that the $i$ th rank $r_{i}=x_{i}-y_{i}$ lies in the interval $[-r+2, M-r-2]$ and furthermore that it satisfies (9).

We first verify that for $1 \leq i \leq l, x_{i} \geq 1$ and $y_{i} \geq 1$. If $\alpha_{i} \equiv r(\bmod 2)$, then using (11) and condition (i),

$$
x_{i}=\left(-r+2 c_{\alpha}(i)+\alpha_{i}+2\right) / 2>\left(-r+2 c_{\alpha}(i)+\left|-2 c_{\alpha}(i)+r-1\right|+2\right) / 2 \geq 1 / 2
$$

and

$$
y_{i}=\left(\alpha_{i}+r-2 c_{\alpha}(i)\right) / 2>\left(\left|2 c_{\alpha}(i)-r+1\right|+r-2 c_{\alpha}(i)\right) / 2 \geq 1 / 2 .
$$

Similarly, if $\alpha_{i} \not \equiv r(\bmod 2)$,

$$
x_{i}=\left(-r+2 c_{\alpha}(i)+\alpha_{i}+1\right) / 2>\left(-r+2 c_{\alpha}(i)+\left|-2 c_{\alpha}(i)+r\right|+1\right) / 2 \geq 1 / 2
$$

and

$$
y_{i}=\left(\alpha_{i}+r-2 c_{\alpha}(i)+1\right) / 2>\left(\left|2 c_{\alpha}(i)-r\right|+r-2 c_{\alpha}(i)+1\right) / 2 \geq 1 / 2 .
$$

Checking the rank of the $i$ th angle

$$
x_{i}-y_{i}=2 x_{i}-\alpha_{i}-1=\left\{\begin{array}{ll}
-r+2 c_{\alpha}(i)+1 & \text { if } \alpha_{i} \equiv r(\bmod 2) \\
-r+2 c_{\alpha}(i) & \text { otherwise }
\end{array},\right.
$$

which satisfies (9).

Now we verify that for $1 \leq i<l, x_{i}>x_{i+1}$ and $y_{i}>y_{i+1}$. We check each of three cases using (11) and condition (ii) of the theorem. If $\alpha_{i} \equiv \alpha_{i+1}(\bmod 2)$, then

$$
\begin{aligned}
& x_{i}-x_{i+1}=\left(\alpha_{i}-\alpha_{i+1}+2\left(c_{\alpha}(i)-c_{\alpha}(i+1)\right)\right) / 2 \geq 1, \\
& y_{i}-y_{i+1}=\left(\alpha_{i}-\alpha_{i+1}-2\left(c_{\alpha}(i)-c_{\alpha}(i+1)\right)\right) / 2 \geq 1 .
\end{aligned}
$$


If $\alpha_{i} \not \equiv \alpha_{i+1} \equiv r(\bmod 2)$, then

$$
\begin{aligned}
& x_{i}-x_{i+1}=\left(\alpha_{i}-\alpha_{i+1}+2\left(c_{\alpha}(i)-c_{\alpha}(i+1)\right)-1\right) / 2 \geq 1, \\
& y_{i}-y_{i+1}=\left(\alpha_{i}-\alpha_{i+1}-2\left(c_{\alpha}(i)-c_{\alpha}(i+1)\right)+1\right) / 2 \geq 1 .
\end{aligned}
$$

Finally, if $\alpha_{i+1} \not \equiv \alpha_{i} \equiv r(\bmod 2)$, then

$$
\begin{aligned}
& x_{i}-x_{i+1}=\left(\alpha_{i}-\alpha_{i+1}+2\left(c_{\alpha}(i)-c_{\alpha}(i+1)\right)+1\right) / 2 \geq 1, \\
& y_{i}-y_{i+1}=\left(\alpha_{i}-\alpha_{i+1}-2\left(c_{\alpha}(i)-c_{\alpha}(i+1)\right)-1\right) / 2 \geq 1 .
\end{aligned}
$$

The following result is an immediate consequence of Theorem 3 and (8).

\section{Corollary 2}

$$
\sum_{n=0}^{\infty}\left|C_{n}(M, r)\right| q^{n}=\frac{1}{(q)_{\infty}} \sum_{j=-\infty}^{\infty}(-1)^{j} q^{j[(M) j+M-2 r] / 2}=\prod_{n \neq 0, \pm r(\bmod M)}\left(1-q^{n}\right)^{-1}
$$

See Figure 2 for an example of the bijection when $M=7, r=1$, and $n=10$. Figure 3 shows the bijection when $M=8, r=3$, and $n=10$.

\section{Remarks on Alternative Colorings}

An alternative coloring is to color the part $\alpha_{i}$, derived from the $i$ th angle with rank $r_{i}$, by:

$$
c_{\alpha}^{\prime}(i)= \begin{cases}r_{i}-k+r & \text { if } r_{i}>k-r \\ -r_{i}+k-r & \text { otherwise }\end{cases}
$$

and proceed to formulate the conditions required to make this mapping a bijection between $(k-1)$-color partitions of $n$ which satisfy the conditions and the set $A_{n}(M, r)$ of partitions of $n$ with all ranks in $[-r+2, M-r-1]$. This is the coloring which would follow by applying coloring ideas in Agarwal and Andrews (for other families) AA to the partition family $A_{n}(M, r)$. This same multi-coloring, $c_{\alpha}^{\prime}$, arises by applying the coloring scheme in Agarwal and Bressoud [AB] (where it was used on a different family) to the lattice path interpretation of $A_{n}(M, r)$ described in Bressoud [Br3], namely, for each peak of height $y$ at location $x$ in the lattice path, create a part of size $x$ with color $y$. This can be shown to be equivalent to the coloring (12) using the bijection between the lattice path and rank interpretations described by Bressoud in [B]3]. An advantage of our coloring is that the color depends only on $r$ and $r_{i}$ and not on $k$. This may make it easier to establish a direct connection between the multi-sum and our multi-color interpretation. The hope is that for our particular goals, this interpretation will be more fruitful in the Lie algebra setting. 


\section{Finitized Rogers-Ramanujan Identities}

In view of (5) and the second equality in (8), the generalized Rogers-Ramanujan identities can be written as

$$
\frac{1}{(q)_{\infty}} \sum_{j=-\infty}^{\infty}(-1)^{j} q^{j[(M) j+M-2 r] / 2}=\sum_{n_{1} \geq \ldots \geq n_{k-1} \geq 0} \frac{q^{n_{1}^{2}+n_{2}^{2}+\ldots+n_{k-1}^{2}+n_{r}+n_{r+1}+\ldots+n_{k-1}}}{(q)_{n_{1}-n_{2}} \ldots(q)_{n_{k-2}-n_{k-1}}\left(q^{2-s} ; q^{2-s}\right)_{n_{k-1}}}
$$

where $s=0$ if $M$ is even and $s=1$ if $M$ is odd. In fact, this is the form of interest in recent work relating Rogers-Ramanujan identities to applications in statistical mechanics and conformal field theory. In these applications, the left-hand side is the bosonic form and the right-hand side is the fermionic form. In the general case, the bosonic form is associated with a character of the minimal model of a Virasoro algebra. In [LW3 and later in [LP] a generalized fermionic Pauli exclusion principle was discovered and discussed in connection with higher level representations of the affine Lie algebra $\widehat{\mathfrak{s l}}_{2}$. Using similar ideas the sum side of the generalized Rogers-Ramanujan identities has recently been studied in several papers, and it has been shown that under certain restrictions, every bosonic form has a fermionic form and, even more, there is a corresponding finitization BMS.

In [FQ], motivated by these connections to physics, Foda and Quano derived finite approximations of the identities (13). We will show that the multi-color partition interpretation also provides an interpretation of the left-hand side for the finitization in this case.

We first make an observation. For $0<r \leq M / 2$ and $u, v \geq 0$, let $F_{n}(M, r, u, v)$ be the set of partitions of $n$ with all ranks in the interval $[-r+2, M-r-2]$ and whose Ferrer diagrams are contained in a $v \times u$ rectangle.

Lemma 3 For $0<r \leq M / 2$ and $u, v \geq 0$, the set $F_{n}(M, r, u, v)$ is in one-to-one correspondence with the set of $k-1$-color partitions $\left(\alpha, c_{\alpha}\right)$ of $n$ in $C_{n}(M, r)$ with the following additional constraint

$$
(i v)(u+v-1)-\alpha_{1} \geq\left|2 c_{\alpha}(1)-r+(v-u)+\frac{1}{2}\right|-\frac{1}{2} .
$$

Proof. Under our map in the proof of Theorem 3, the partitions in $F_{n}(M, r, u, v)$ are mapped into $k-1$ color partitions $\left(\alpha_{i}\right)$ satisfying (i), (ii), and (iii) in Theorem 3. Since this map was shown to be a bijection, it suffices to show that its image is characterized by the extra condition (iv). So, for a $k-1$ color partition $\alpha$ satisfying (i)-(iii), we derive necessary and sufficient conditions on $\alpha$ to guarantee that under the inverse mapping its first angle has width $x_{1} \leq u$ and height $y_{1} \leq v$. The inverse map (11) has

$$
x_{1}=\left\lfloor\left(-r+2 c_{\alpha}(1)+\alpha_{1}+2\right) / 2\right\rfloor
$$


so $x_{1} \leq u$ is equivalent to

$$
\frac{-r+2 c_{\alpha}(1)+\alpha_{1}+1}{2} \leq u
$$

which in turn is equivalent to

$$
2 c_{\alpha}(1)-r+(v-u) \leq(u+v-1)-\alpha_{1} .
$$

On the other hand, the height $y_{1}=\alpha_{1}-x_{1}+1$ must satisfy $y_{1} \leq v$ which is equivalent to $x_{1} \geq-v+\alpha_{1}+1$, so we require

$$
\frac{-r+2 c_{\alpha}(1)+\alpha_{1}+2}{2} \geq-v+\alpha_{1}+1
$$

which is equivalent to

$$
-2 c_{\alpha}(1)+r-(v-u)-1 \leq(u+v-1)-\alpha_{1} .
$$

Combining (14) and (15) gives the lemma.

In [FQ], Foda and Quano derive the following finitized Rogers-Ramanujan identity:

$$
\begin{aligned}
\sum_{j=-\infty}^{\infty} & (-1)^{j} q^{j[(2 k+1) j+2 k+1-2 r] / 2}\left[\begin{array}{c}
N \\
\left\lfloor\frac{N-k+r-(2 k+1) j}{2}\right\rfloor
\end{array}\right]_{q} \\
= & \sum q^{n_{1}^{2}+\cdots+n_{k-1}^{2}+n_{r}+\cdots+n_{k-1}} \prod_{j=1}^{k-1}\left[\begin{array}{c}
N-2\left(n_{1}+\cdots+n_{j-1}\right)-n_{j}-n_{j+1}-a_{r j}^{(k)} \\
n_{j}-n_{j+1}
\end{array}\right]_{q},
\end{aligned}
$$

where the sum runs over $n_{1} \geq \cdots \geq n_{j}=0$ such that $2\left(n_{1}+\cdots+n_{k-1}\right) \leq N-k+r$ and $a_{i j}^{(k)}$ is the $i j$-entry of the $k \times(k-1)$ matrix

$$
A^{(k)}=\left(\begin{array}{cccc}
1 & 2 & \cdots & k-1 \\
0 & 1 & \cdots & k-2 \\
\vdots & \vdots & \ddots & \vdots \\
0 & 0 & \cdots & 1 \\
0 & 0 & \cdots & 0
\end{array}\right) .
$$

When $N \rightarrow \infty$, the identity reduces to (13) in the case of odd $M$ and $s=1$.

Foda and Quano prove that the left-hand side of (16) is the generating function for the set of partitions $F_{n}(M, r, u, v)$ in the special case that $M=2 k+1, u=\lfloor(N+k-r+1) / 2\rfloor$, and $v=\lfloor(N-k+r) / 2\rfloor$. Combining this with Lemma (3) we get the following.

Corollary 3 For $M=2 k+1$ and $r \leq k$, the left-hand side of (10) is the generating function for the set of $k-1$-color partitions $\left(\alpha, c_{\alpha}\right)$ of $n$ in $C_{n}(M, r)$ with the following additional constraint

$$
\text { (iv) }(N-1)-\alpha_{1} \geq \begin{cases}\left|2 c_{\alpha}(1)-k+\frac{1}{2}\right|-\frac{1}{2}, & \text { if } N+k \equiv r(\bmod 2) \\ \left|2 c_{\alpha}(1)-k-\frac{1}{2}\right|-\frac{1}{2}, & \text { otherwise }\end{cases}
$$

In particular, $\alpha_{1} \leq N-1$. 
In [FQ], Foda and Quano derive the following additional finitized Rogers-Ramanujan identity:

$$
\begin{array}{r}
\sum_{j=-\infty}^{\infty}(-1)^{j} q^{j(k j+k-r)}\left[\begin{array}{c}
2 N+k-r \\
N-k j
\end{array}\right]_{q}=\sum q^{n_{1}^{2}+\cdots+n_{k-1}^{2}+n_{r}+\cdots+n_{k-1}} \times \\
\prod_{j=1}^{k-2}\left[\begin{array}{c}
2 N-2\left(n_{1}+\cdots+n_{j-1}\right)-n_{j}-n_{j+1}-b_{r j}^{(k)} \\
n_{j}-n_{j+1}
\end{array}\right]_{q}\left[\begin{array}{c}
N-\left(n_{1}+\cdots+n_{k-2}\right) \\
n_{k-1}
\end{array}\right]_{q^{2}},
\end{array}
$$

where the sum runs over $n_{1} \geq \cdots \geq n_{j}=0$ such that $n_{1}+\cdots+n_{k-1} \leq N$ and $b_{i j}^{(k)}$ is the $i j$-entry of the $k \times(k-2)$ matrix

$$
B^{(k)}=\left(\begin{array}{ccccc}
k-2 & k-3 & k-4 & \cdots & 1 \\
k-2 & k-3 & k-4 & \cdots & 1 \\
k-3 & k-4 & k-5 & \cdots & 1 \\
\vdots & \vdots & \vdots & \cdots & \vdots \\
3 & 2 & 1 & \cdots & 1 \\
2 & 1 & 1 & \cdots & 1 \\
1 & 1 & 1 & \cdots & 1 \\
0 & 0 & 0 & \cdots & 0
\end{array}\right) .
$$

When $N \rightarrow \infty$, the identity reduces to (13) in the case of even $M$ and $s=0$.

Foda and Quano prove that the left-hand side of (17) is the generating function for the set of partitions $F_{n}(M, r, u, v)$ in the special case that $M=2 k, u=N+k-r$, and $v=N$. Combining this with Lemma 3 we get:

Corollary 4 For $M=2 k$, the left-hand side of $(17)$ is the generating function for the set of $k-1$-color partitions $\left(\alpha, c_{\alpha}\right)$ of $n$ in $C_{n}(M, r)$ and the following additional constraint

$$
\text { (iv) }(2 N+k-r-1)-\alpha_{1} \geq\left|2 c_{\alpha}(1)-k+\frac{1}{2}\right|-\frac{1}{2} .
$$

Remark 3 Clearly, our map in Theorem 5 , when restricted to partitions in $F_{n}(M, r, u, v)$ with Durfee square of size $d$, gives a bijection with those $k-1$ color partitions of $n$ in $C_{n}(M, r)$ which have exactly d parts and satisfy (iv) of of Lemma 3 .

Acknowledgment We would like to thank James Lepowsky for helpful comments on an earlier version of this paper and Christian Krattenthaler for bringing the references [AA] and $\mathrm{AB}$ to our attention. 


$\begin{array}{lll}\begin{array}{l}\text { Partition } \pi \\ \text { of } 10 \text { with all } \\ \text { ranks in }[1,4]\end{array} & \begin{array}{l}\text { rank vector } \\ \text { of } \pi\end{array} & \begin{array}{l}\text { the 2-color partition } \\ \left(\alpha(\pi), c_{\alpha(\pi)}\right) \text { of } 10\end{array} \\ (7,1,1,1) & {[3]} & \left(10_{2}\right) \\ (6,4) & {[4,2]} & \left(7_{2}, 3_{1}\right) \\ (6,3,1) & {[3,1]} & \left(8_{2}, 2_{1}\right) \\ (6,1,1,1) & {[1]} & \left(10_{1}\right) \\ (5,5) & {[3,3]} & \left(6_{2}, 4_{2}\right) \\ (5,4,1) & {[2,2]} & \left(7_{1}, 3_{1}\right) \\ (5,3,1,1) & {[1,1]} & \left(8_{1}, 2_{1}\right) \\ (4,4,2) & {[1,1]} & \left(6_{1}, 4_{1}\right)\end{array}$

Figure 2: Example of the bijection of Theorem 3 when $M=7, r=1$, and $n=10$.

$\begin{array}{lll}\begin{array}{l}\text { Partition } \pi \\ \text { of } 10 \text { with all } \\ \text { ranks in }[-1,3]\end{array} & \begin{array}{l}\text { rank vector } \\ \text { of } \pi\end{array} & \begin{array}{l}\text { the 3-color partition } \\ \left(\alpha(\pi), c_{\alpha(\pi)}\right) \text { of } 10\end{array} \\ (6,2,1,1) & {[2,0]} & \\ (6,1,1,1,1) & {[1]} & \left(9_{2}, 1_{1}\right) \\ (5,4,1) & {[2,2]} & \left(10_{2}\right) \\ (5,3,2) & {[2,0]} & \left(7_{2}, 3_{2}\right) \\ (5,3,1,1) & {[1,1]} & \left(7_{2}, 3_{1}\right) \\ (5,2,2,1) & {[1,-1]} & \left(8_{2}, 2_{2}\right) \\ (5,2,1,1,1) & {[0,0]} & \left(8_{2}, 2_{1}\right) \\ (5,1,1,1,1,1) & {[-1]} & \left(9_{1}, 1_{1}\right) \\ (4,4,2) & {[1,1]} & \left(6_{1}\right) \\ (4,4,1,1) & {[0,2]} & \left(7_{1}, 3_{2}\right) \\ (4,3,3) & {[1,0,0]} & \left(6_{2}, 3_{1}, 1_{1}\right) \\ (4,3,2,1) & {[0,0]} & \left(7_{1}, 3_{1}\right) \\ (4,3,1,1,1) & {[-1,1]} & \left(8_{1}, 2_{2}\right) \\ (4,2,2,1,1) & {[-1,-1]} & \left(8_{1}, 2_{1}\right) \\ (3,3,3,1) & {[-1,0,0]} & \left(6_{1}, 3_{1}, 1_{1}\right) \\ (3,3,2,2) & {[-1-1]} & \left(6_{1}, 4_{1}\right) \\ (7,1,1,1) & {[3]} & \left(10_{3}\right) \\ (6,3,1) & {[3,1]} & \left(8_{3}, 2_{2}\right) \\ (6,2,2) & {[3,-1]} & \left(8_{3}, 2_{1}\right) \\ (5,5) & {[3,3]} & \left(6_{3}, 4_{3}\right)\end{array}$

Figure 3: Example of the bijection of Theorem a 3 when $M=8, r=3$, and $n=10$. 


\section{References}

[AA] A. K. Agarwal and G. E. Andrews, Rogers-Ramanujan identities for partitions with $N$ copies of $N$, J. Combin. Theory Ser. A 45 (1987), 40-49.

[AB] A. K Agarwal and D. M. Bressoud, Lattice paths and multiple basic hypergeometric series, Pacific J. Math. 136, No. 2 (1989), 209-228.

[An1] G. E. Andrews, The theory of partitions, Encyclopedia of Mathematics and its applications (Rota ed.), 2, Addison-Wesley, Reading, 1976.

[An2] G. E. Andrews, q-Series: their development and application in analysis, number theory, combinatorics, physics, and computer algebras, CBMS Regional Conf. Ser. in Math. 66, Amer. Math. Soc., Providence, RI, 1986.

[An3] G. E. Andrews, Sieves in the theory of partitions, American J. Math. 94 (1972), 1214-1230.

[An4] G. E. Andrews, Partitions and Durfee dissection, American J. Math. 101 (1979), 735-742.

[BeM] A. Berkovich and B. M. McCoy, Rogers-Ramanujan identities: a century of progress from mathematics to physics, Proceedings of the International Congress of Mathematicians, Vol. III (Berlin, 1998), Doc. Math. 1998, Extra Vol. III, 163-172 (electronic).

[BMS] A. Berkovich, B. McCoy and A. Schilling, Rogers-Schur-Ramanujan type identities for the $M\left(p, p^{\prime}\right)$ minimal models of conformal field theory. Comm. Math. Phys. 191 (1998), 325-395.

[Br1] D. M. Bressoud, A generalization of the Rogers-Ramanujan identities for all moduli, J. Combin. Theory Ser. A 27 (1979), 64-68.

[Br2] D. M. Bressoud, Extension of the partition sieve, J. Number Theory 12 (1980), $87-100$.

[Br3] D. M. Bressoud, Lattice paths and the Rogers-Ramanujan identities, Number Theory, Madras 1989, 140-172, Lecture Notes in Math. 1395, Springer, Berlin-New York 1989.

[Bu1] W. H. Burge, A correspondence between partitions related to generalizations of the Rogers-Ramanujan identities, Discrete Math. 34 (1981), 9-15.

[Bu2] W. H. Burge, A three-way correspondence between partitions, European J. Combinatorics 3 (1982), 195-213. 
[BM] M. Bos and K.C. Misra, Level two representations of $A_{7}^{(2)}$ and Rogers-Ramanujan identities, Comm. Algebra 22 (1994), 3965-3983.

[FS] B. Feigin and A. Stoyanovskii, Functional models of the representations of current algebras, and semi-infinite Schubert cells, Funct. Anal. Appl. 28 (1994), no. 1, 55-72; Quasi-particles model for the representations of Lie algebras and geometry of flag manifolds, hep-th/9308079).

[FQ] O. Foda and Y.-H. Quano, Polynomial identities of the Rogers-Ramanujan type, Int. J. Mod. Phys. A 10 (1995), 2291-2315.

[G] B. Gordon, A combinatorial generalization of the Rogers-Ramanujan identities, Amer. J. Math. 83 (1961), 393-399.

[Ge] G. Georgiev, Combinatorial constructions of modules for infinite-dimensional Lie algebras, I. Principal subspace. J. Pure Appl. Algebra 112 (1996), 247-286; II. Parafermionic space, Q-alg/9504024.

$[\mathrm{LM}]$ J. Lepowsky and S. Milne, Lie algebraic approaches to classical partition identities, Adv. in Math. 29 (1978), 15-59.

[LP] J. Lepowsky and M. Primc, Structure of the standard modules for the affine Lie algebra $A_{1}^{(1)}$. Cont. Math., 46. Amer. Math. Soc., 1985.

[LW1] J. Lepowsky and R.L. Wilson, Construction of the affine Lie algebra $A_{1}^{(1)}$, Comm. Math. Phys. 62 (1978), 43-53.

[LW2] J. Lepowsky and R.L. Wilson, A Lie theoretic interpretation and proof of the RogersRamanujan identities, Adv. in Math. 45 (1982), 21-72.

[LW3] J. Lepowsky and R.L. Wilson, A new family of algebras underlying the RogersRamanujan identities and generalizations, Proc. Nat. Acad. Sci. U.S.A. 78 (1981), $7254-7258$.

[LW4] J. Lepowsky and R.L. Wilson, The structure of standard modules, I: Universal algebras and the Rogers-Ramanujan identities, Invent. Math. 77 (1984), 199-290; II, The case $A_{1}^{(1)}$, principal gradation, Invent. Math. 79 (1985), 417-442.

[Ma] M. Mandia, Structure of the level one standard modules for the affine Lie algebras $B_{\ell}^{(1)}, F_{4}^{(1)}$, and $G_{2}^{(1)}$, Mem. Amer. Math. Soc. 65 (1987).

[M1] K.C. Misra, Structure of certain standard modules for $A_{n}^{(1)}$ and the RogersRamanujan identities, J. Algebra 88 (1984), 196-227.

[M2] K.C. Misra, Structure of some standard modules for $C_{n}^{(1)}$, J. Algebra 90 (1984), 385-409. 
[M3] K.C. Misra, Realization of the level two standard $s \ell(2 k+1, \mathbb{C})^{\sim}$-modules, Trans. Amer. Math. Soc. 316 (1989), 295-309.

[MP1] A. Meurman and M. Primc, Annihilating ideals of standard modules of $\operatorname{s\ell }(2, \mathbb{C})^{\sim}$ and combinatorial identities, Adv. in Math. 64 (1987), 177-240.

[MP2] A. Meurman and M. Primc, Annihilating fields of standard modules of $s \ell(2, \mathbb{C})^{\sim}$ and combinatorial identities, Memoirs Amer. Math. Soc. 652 (1999).

[MP3] A. Meurman and M. Primc, A basis of the basic $s \ell(3, \mathbb{C})^{\sim}$-modules, math.QA/9812029.

[P] M. Primc, Some crystal Rogers-Ramanujan type identities, Glas. Mat. Ser. III 34(54) (1999), 73-86.

[X] C. Xie, Structure of the level two standard modules for the affine Lie algebra $A_{2}^{(2)}$, Comm. Algebra 18 (1990), 2397-2401. 